Schmerz 2009 $\cdot 23: 448-460$

DOI 10.1007/s00482-009-0819-0

Online publiziert: 11. Juli 2009

(c) Deutsche Gesellschaft zum Studium des Schmerzes. Published by Springer

Medizin Verlag - all rights reserved 2009

\author{
J.C. Türp ${ }^{1}$ A. Hugger ${ }^{2} \cdot$ C. Löst ${ }^{3} \cdot$ P. Nilges ${ }^{4} \cdot$ H.J. Schindler ${ }^{5}$ H.J. Staehle ${ }^{6}$ \\ ${ }^{1}$ Klinik für Rekonstruktive Zahnmedizin und Myoarthropathien, \\ Universitätskliniken für Zahnmedizin, Universität Basel \\ ${ }^{2}$ Poliklinik für Zahnärztliche Prothetik, Westdeutsche Kieferklinik, \\ Universität Düsseldorf \\ ${ }^{3}$ Poliklinik für Zahnerhaltung, Zentrum für Zahn-, Mund- und Kieferheilkunde, \\ Universität Tübingen \\ ${ }^{4}$ DRK-Schmerzzentrum Mainz \\ ${ }^{5}$ Poliklinik für Zahnärztliche Prothetik, Klinik für Mund-, Zahn- und Kieferkrankheiten, \\ Universitätsklinikums Heidelberg \\ ${ }^{6}$ Poliklinik für Zahnerhaltungskunde, Klinik für Mund-, Zahn- und Kieferkrankheiten, \\ Universitätsklinikum Heidelberg
}

\title{
Vorschlag einer Klassifikation der Odontalgien
}

\section{Hintergrund und Ziel}

Aus der bislang größten bevölkerungsrepräsentativen Erhebung über die Verbreitung von orofazialen Schmerzen in den USA - sie wurde im Jahre 1989 durchgeführt - lässt sich entnehmen, dass Odontalgien in ihrer Häufigkeit alle anderen orofazialen Schmerzen überragen: 12,2\% (Frauen: 12,5\%; Männer: 12,0\%) von 42.370 teilnehmenden Erwachsenen hatten angegeben, während der vergangenen 6 Monate unter Zahnschmerzen gelitten zu haben [43]. Dabei war die Prävalenz in der Altersgruppe zwischen 18 und 34 Jahren mit $17,0 \%$ am höchsten, gefolgt von $12,1 \%$ bei den 35 - bis 54 -Jährigen, $6,8 \%$ bei den 55 - bis 74 -Jährigen und $3,4 \%$ bei den über 74-Jährigen.

Daher überrascht es nicht, dass der Großteil der mit orofazialen Schmerzen eine Zahnarztpraxis aufsuchenden Patienten zahnbezogene Beschwerden hat. Erst an zweiter Stelle folgen Personen mit Schmerzen in der Kaumuskulatur und/ oder den Kiefergelenken (vgl. [38]). Dabei ist die Diagnosestellung oft alles andere als einfach.

Zahnschmerz löst bei den meisten Betroffenen unmittelbare psychische, soziale und verhaltensbezogene Wirkungen aus. Dazu zählen Konzentrationsprobleme, Behinderungen bei der häuslichen Arbeit und in der Freizeit, Vermeidung bestimmter Nahrungsmittel bzw. Speisen, Einnahme von Analgetika und Aufsuchen eines Zahnarztes [24, 45]. Die Einschränkungen und Auffälligkeiten werden - als Faustregel - umso deutlicher, je stärker der Zahnschmerz ausgeprägt ist [45].

Die außerordentliche Vielgestaltigkeit der Odontalgien, ihre beachtliche Bevölkerungsprävalenz, die äußerst unangenehme Schmerzqualität und -intensität sowie ihre psycho-sozial-behavioralen Auswirkungen auf die davon betroffene Person lassen es wünschenswert erscheinen, sich einer Zahnschmerzeinteilung bedienen zu können, die eine rasche Diagnosefindung erlaubt. Eine verbreitete zeitgenössische Einteilung der Odontalgien ist diejenige der Internationalen $\mathrm{Ge}$ sellschaft zum Studium des Schmerzes (IASP) aus dem Jahre 1994 [50]. In ihr werden folgende vier Zahnschmerzformen unterschieden:

- Dentinschmerz, bedingt durch Schmelz-Dentin-Defekte,
- Pulpaschmerz, hervorgerufen durch eine Pulpitis,

- Schmerzen in Zusammenhang mit einer apikalen Parodontitis bzw. einem periapikalen Abszess,

- „nicht mit Läsionen in Zusammenhang stehende Zahnschmerzen“" (atypische Odontalgie).

Detaillierter ist die Taxonomie von Okeson [56], in der wie folgt differenziert wird:

- Zahnschmerzen pulpalen Ursprungs (akut, chronisch, rezidivierend, Pulpaschmerz mit parodontalen Elementen),

- Zahnschmerzen parodontalen Ursprungs,

- Zahnschmerz aufgrund gestörter zentraler Inhibition,

- Zahnschmerzen nichtdentalen Ursprungs (muskulär, neurovaskulär, kardiogen, neuropathisch, sinugen/ rhinogen, psychogen).

Tronstad [77] unterscheidet zwischen pulpalen und periapikalen Schmerzen und fasst in einer 3. Gruppe „orale und periorale Schmerzen von endodontischem Interesse“ zusammen, allerdings ohne Letz- 
Hier steht eine Anzeige.

算 Springer 
terer ein Ordnungsprinzip zugrunde zu legen. Gleiches gilt für Thoden van Velzen et al. [74].

Für die klinische Arbeit am Patienten sind jedoch durchgehend strukturierte Klassifikationen vorteilhaft. Ziel der vorliegenden Arbeit war daher, eine verfeinerte Einteilung der Odontalgien zu erarbeiten, die alle bekannten Zahnschmerzformen aufgreift und ihre jeweiligen diagnostischen Merkmale so zusammenfasst, dass im klinischen Alltag eine rasche „Mustererkennung“ ([25]; und damit Diagnosestellung) möglich ist.

\section{Methode}

In einem nominalen Gruppenprozess wurde von H.J. Schindler (Karlsruhe/Heidelberg), H.J. Staehle (Heidelberg) und J.C. Türp (Basel) im Jahre 2002 auf der Basis der IASP-Klassifikation von 1994 eine verfeinerte Einteilung der Odontalgien erarbeitet. Dabei sollte, sofern möglich, jede Art der Odontalgie anhand folgender klinischer Kriterien beschrieben werden:

- Ursache,

- Dauer,

- Qualität,

- Intensität (jeweils unterschieden in „schwach“, „mittelstark“, ,stark“, „sehr stark"),

- Auslösung,

- Reaktion auf Sensibilitätstest,

- Reaktion auf Perkussionstest,

- Befund in bildgebenden Verfahren,

- Sonderform(en),

- Therapie.

Das Ergebnis dieser Bemühungen wurde 2003 auf einer wissenschaftlichen Tagung erstmals präsentiert [79]. Periodische Überprüfungen der Klassifikation und ihrer Inhalte führten zu Modifikationen des ursprünglichen Klassifikationsentwurfs, die in einem fortgesetzten nominalen Gruppenprozess unter Einbeziehung von A. Hugger, C. Löst und P. Nilges schließlich in die 2009 fertiggestellte und hier vorgestellte Fassung mündeten.

\section{Ergebnisse}

Die erarbeitete Klassifikation unterscheidet folgende 7 mit Zähnen assoziierte Schmerzformen (• Tab. 1):
1. „Dentinschmerz", ausgehend von gesundem oder „reversibel“ (im Initialstadium) entzündetem Pulpagewebe,

2. „Pulpaschmerz", ausgehend von „reversibel“ (im fortgeschrittenen Stadium) oder ,irreversibel“ entzündetem Pulpagewebe,

3. parodontaler Schmerz,

4. alveolär-ossärer Schmerz,

5. Formenkreis der atypischen Zahnschmerzen (atypische Odontalgie/ Phantomzahnschmerz),

6. in die Zähne übertragener (heterotoper) Schmerz,

7. Zahnschmerz in Zusammenhang mit primär psychosozialen Faktoren.

\section{Diskussion}

Im Vergleich zur IASP-Version [50] unterscheidet die hier vorgestellte Klassifikation der Odontalgien 7 Zahnschmerzformen, die zum Teil wiederum untergliedert werden können (• Tab. 1).

\section{1. „,Dentinschmerz"}

An dem durch mechanische Reize ausgelösten Dentinschmerz sind vorwiegend A$\delta$-Fasern beteiligt; C-Fasern werden demgegenüber unter anderem durch potenziell schädigende thermische Reize erregt. Da alle bisherigen Erklärungsversuche zur Reizweiterleitung im Dentin (z. B. [4, 5, 6, 7]) Unzulänglichkeiten aufweisen, ist die derzeit am weitesten verbreitete Theorie relativ unspezifisch: Eine Verschiebung des Inhalts der Dentinkanälchen führt in der Pulpa zu einer Volumenverschiebung bzw. Druckänderung, wodurch „mechanosensitive Nervenstrukturen im Prädentin oder sogar in der darunterliegenden Pulpa" angeregt werden [57].

Es lassen sich vier Arten des „Dentinschmerzes" differenzieren:

\subsection{Physiologische Sensibilität}

Die physiologische Variante wird durch thermische (sehr kalte oder sehr heiße) und elektrische Reize ausgelöst. Die durch sie ausgelöste Schmerzreaktion macht man sich bei der zahnbezogenen Diagnostik in Form der thermischen oder auch elektrischen Sensibilitätstestung zunutze, deren Ergebnis Rückschlüsse auf eine intakte Reizantwort der Zahnpulpa gibt [6o].

Andere Reize für einen „physiologischen" Dentinschmerz sind vorwiegend mechanischer Art, z. B. bei der Zahnsteinentfernung von den Zahnwurzeloberflächen mit Handinstrumenten [48] oder Ultraschall $[8,37]$. Die Pulpa ist - im Gegensatz zum parodontalen Gewebe - ein nozizeptives Organ, das neben Schmerzreizen keine anderen Empfindungen zulässt.

\subsection{Präparationsschmerz und Dentinüberempfindlichkeit}

Auch der Zahnschmerz, den ein Patient durch Beschleifen der Zahnhartsubstanzen (Schmelz, Dentin) während zahnärztlicher Therapiemaßnahmen (bei unzureichend wirkender oder fehlender Lokalanästhesie) spürt, ist ein "physiologischer Dentinschmerz".

Eine besondere Herausforderung stellt für Zahnärzte bis heute die Dentinhypersensibilität dar, v. a. in Form überempfindlicher Zahnhälse. Trotz großer Anstrengungen konnte bislang kein zufriedenstellendes Behandlungsmittel gefunden werden [9]. Dentinhypersensibilitäten können sich auch nach zahnärztlichen Eingriffen ergeben, z. B. nach Scaling und/oder Wurzelglättung.

\subsection{Postoperative (auch postrestaurative) Hypersensibilität}

Vor allem seit der Einführung der adhäsiven Befestigung von Einzelzahnrestaurationen trat das Phänomen der „postoperativen Hypersensibilität“ auf. Nach (unerwünschter) Spaltbildung zwischen Dentin und Adhäsiv resultieren - so vermutet man - schon bei physiologischer Kaubewegung Pumpeffekte, die zu einer schnellen Flüssigkeitsbewegung in den Dentintubuli führen sollen $[5,6,7]$. Eine spontane Rückbildung durch Verengung bzw. Verschluss der betroffenen Dentintubuli ist möglich. Ansonsten empfiehlt sich ein Austausch der Restauration.

\subsection{Infraktur}

Gut $\mathrm{zu}$ therapieren, aber oft schwierig $\mathrm{zu}$ diagnostizieren ist demgegenüber ei- 
ne Infraktur (Infraktion) [12, 46, 63, 78]. Sie ist durch Risse oder unvollständige Frakturen gekennzeichnet, die durch den Schmelz in das Dentin reichen. Im Falle einer pulpanahen Infraktion ist das Leitsymptom der kurze, stechende Aufbiss- bzw. Entlastungsschmerz. Der Entlastungsschmerz, ein kurz dauernder Schmerz nach Beißen auf harte Nahrung oder einen harten Gegenstand (Holzspatel; Plastikgriff), ist nicht immer gegeben; wenn er auftritt, gilt er als Leitsymptom für eine Infraktur [12].

\section{2. „Pulpaschmerz"}

Der „Pulpaschmerz“ wird vorwiegend durch C-Fasern ausgelöst, deren Reizschwelle durch Entzündungsprozesse verändert wird. Die C-Fasern machen (in Form afferenter und efferenter Fasern) den überwiegenden Anteil der Pulpaneuronen aus [57].

Der akute pulpale Schmerz ist der Inbegriff des „klassischen“ Zahnschmerzes. Dieser dumpfe, tiefe Schmerz [28] ist für den Patienten meist schlecht lokalisierbar und kann von extremer Intensität sein. Der akute „Pulpaschmerz" kann episodisch oder anhaltend auftreten (intermittierender Spontanschmerz); unter besonderen Umständen kann er auch chronifizieren.

Okeson [56] rechnet den vom Pulpagewebe ausgehenden Schmerz den Eingeweideschmerzen zu. In der Tat kann der Pulpaschmerz einige Merkmale viszeraler Schmerzen aufweisen $[26,32]$ :

- Viszerale Schmerzen sind diffus lokalisiert.

- Sie bestehen aus einem Organschmerz und einem Schmerz, der in andere Körperstrukturen übertragen wird. Auch Schmerzen im „Pulpa-Dentin-Organ“" strahlen häufig in benachbarte (weiter distal gelegene) Zähne, in Schläfen, die Ohren- oder die Wangenregion aus.

- Sie sind mit negativen affektiven Empfindungen verbunden.

- Sie werden von somatomotorischen und vegetativen Reaktionen begleitet.

Nach derzeitigem neurophysiologischem Wissen sowie aufgrund entwicklungsgeschichtlicher Gesichtspunkte und Ana-

Schmerz 2009 - 23:448-460 DOI 10.1007/s00482-009-0819-0

(c) Deutsche Gesellschaft zum Studium des Schmerzes.

Published by Springer Medizin Verlag - all rights reserved 2009

\section{J.C. Türp · A. Hugger · C. Löst · P. Nilges · H.J. Schindler · H.J. Staehle Vorschlag einer Klassifikation der Odontalgien}

\section{Zusammenfassung}

Hintergrund. Die Prävalenz von Zahnschmerzen in der Bevölkerung ist beachtlich. Bislang publizierte Klassifikationen der Odontalgien erscheinen jedoch für klinische Belange nicht ausreichend strukturiert. Zudem finden nicht alle bekannten Zahnschmerzformen Berücksichtigung. Ziel dieser Abhandlung ist die Vorstellung einer verfeinerten und aktuellem Wissen genügenden Einteilung der Odontalgien und die Zusammenfassung ihrer jeweiligen Merkmale zum Zweck einer raschen Diagnosestellung. Ergebnisse. Die erarbeitete Klassifikation unterscheidet 7 Schmerzformen: 1. „DentinSchmerz" (ausgehend vom Pulpagewebe), 2. „Pulpa-Schmerz" (ausgehend vom Pulpagewebe), 3. parodontaler Schmerz, 4. alveolär-ossärer Schmerz, 5. atypische Odontalgie,
6. in die Zähne übertragener (heterotoper) Schmerz, 7. Zahnschmerz in Zusammenhang mit primär psychosozialen Faktoren.

Schlussfolgerungen. Die vorgeschlagene Klassifikation erscheint geeignet, die verschiedenen Formen der Odontalgien differenzierter darzustellen, als dies mit den bisherigen Einteilungen der Fall war. Ihre Brauchbarkeit bzw. Überlegenheit gegenüber anderen Klassifikationen wird sich im klinischen Alltag erweisen müssen.

Schlüsselwörter Klassifikationssystem - Odontalgien · Pulpitis . Apikale Parodontitis · Alveolitis · Atypische Odontalgie · Heterotoper Schmerz · "Psychogener" Schmerz - Diagnostik - Therapieempfehlungen

\section{Suggestion for a classification of odontalgias}

\section{Abstract}

Background. Toothache prevalence in the overall population is considerable. However, for clinical purposes, the classification schemes available do not appear to be sufficiently sophisticated. Moreover, not all known forms of dental pain are considered. A refined classification that meets current standards is therefore introduced. To facilitate diagnosis, the characteristic features of the various types of odontalgia are summarized. Results. The new classification differentiates among seven different origins of pain: 1. dentinal pain (originating from the pulpal tissues), 2. pulpal pain (originating from the pulpal tissues), 3. periodontal pain, 4. alveolar-osseous pain, 5. atypical odontalgia,
6. heterotopic dental pain, 7. odontalgia associated with primary psychosocial factors. Conclusions. In our opinion, the proposed classification differentiates among the different forms of odontalgia more precisely than all previous ones. However, its viability and advantages over other available classification schemes still need to be verified in daily practice.

\section{Keywords}

Classification system · Odontalgia · Pulpitis . Apical periodontitis - Alveolitis - Atypical odontalgia · Heterotopic pain · Psychogenic pain - Pain evaluation - Therapeutic recommendation 
Tab. 1 Klassifikation von mit Zähnen assoziierten Schmerzformen (oder Odontalgien)

1.„Dentinschmerz" (ausgehend vom Pulpagewebe bei - angenommen - primär gesunder Pulpa)

\begin{tabular}{|c|c|c|}
\hline \multirow[t]{4}{*}{ Ursache } & 1.1 & $\begin{array}{l}\text { Physiologische Schmerzreaktion auf einen äußeren Reiz (äußerlich intakter Zahn; Pulpitis unwahrscheinlich, } \\
\text { allenfalls,,reversible“ Pulpitis im Initialstadium) } \\
\text { Diagnose: } \\
\text { - Physiologische Sensibilität (im Sinne einer regelgerechten Reizantwort) }\end{array}$ \\
\hline & 1.2 & $\begin{array}{l}\text { Physiologische Schmerzreaktion auf einen äußeren Reiz bei Schmelz-Dentin-Läsionen (freiliegendes Dentin; } \\
\text { gesunde Pulpa oder, „reversible“ Pulpitis im Initialstadium) } \\
\text { Diagnose: } \\
\text { - Dentinüberempfindlichkeit (Dentinhypersensibilität, Dentinhyperästhesie) } \\
\text { Bemerkung: kann zu, ,irreversibler“ Pulpitis führen, dann s. } 2.1\end{array}$ \\
\hline & 1.3 & $\begin{array}{l}\text { Physiologische Schmerzreaktion auf Kaubelastung einer adhäsiv verankerten (oder befestigten) Einzelzahn- } \\
\text { restauration (Spaltbildung zwischen präpariertem Dentin und Adhäsiv; keine Pulpitis) } \\
\text { Diagnose: } \\
\text { Postoperative Hypersensibilität (z. B. nach restaurativen Eingriffen) } \\
\text { Bemerkung: kann in Pulpitis resultieren, dann s. } 2.1\end{array}$ \\
\hline & 1.4 & $\begin{array}{l}\text { Schmerzreaktion auf einen äußeren mechanischen Reiz nach Rissbildung im Dentin (keine „irreversible“ Pulpitis; } \\
\text { kann zu „irreversibler“ Pulpitis und apikaler Parodontitis führen, dann s. 2.1 bzw. 3.2). } \\
\text { Diagnose: } \\
\text { - Infraktur (Infraktion; Cracked-Tooth-Syndrom) }\end{array}$ \\
\hline Dauer & & Kurz (Sekunden/Minuten) \\
\hline Qualität & & 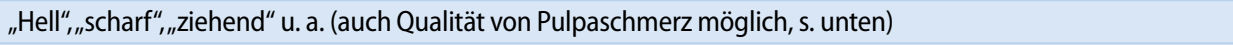 \\
\hline Intensität & & Schwach bis mittelstark abhängig von Reizstärke; 1.2 und 1.4 auch bis sehr stark \\
\hline Auslösung & & $\begin{array}{l}\text { 1.1/1.2: Nur auf einen äußeren Reiz [thermisch (kalt, heiß), elektrisch, mechanisch], nicht spontan } \\
\text { 1.2: Auch auf osmotischen Reiz (süß, sauer) und Luft } \\
\text { 1.3/1.4: Durch Belastung (Aufbiss) oder Entlastung nach vorheriger Belastung des Zahns } \\
\text { (sog. Entlastungsschmerz) }\end{array}$ \\
\hline Sensibilitätstest & & Positiv \\
\hline Perkussionstest & & Negativ \\
\hline Röntgen & & Ggf. Zahnhartsubstanzläsionen sichtbar, pulpaler und periradikulärer Bereich unauffällig \\
\hline Therapie & & $\begin{array}{l}\text { 1.1: Keine; } 1.2 \text { : kausal oder symptomatisch, je nach Läsionsumfang; } 1.3 \text { initial exspektative Diagnostik/kausal; } \\
\text { 1.4 kausal }\end{array}$ \\
\hline
\end{tabular}

\section{2. „Pulpaschmerz" (ausgehend vom Pulpagewebe durch Induzierung einer „reversiblen“ oder „, irreversiblen“ Pulpitis)}

Ursache 2.1

Pathophysiologische Schmerzreaktion bei Entzündung der Pulpa aufgrund von mikrobiellen Reizen, z. B. infolge Karies, Infektionsübertragung aus der Nachbarschaft oder hämatogener (retrograder) Streuung von Bakterien (Anachorese)

Diagnose:

- Pulpitis aufgrund mikrobieller Infektion

2.2 Pathophysiologische Schmerzreaktion bei Entzündung der Pulpa aufgrund von chemischen Reizen [Devitalisationsmittel, z. B. Arsen, (Para-)Formaldehyd]

Diagnose:

- Pulpitis aufgrund chemisch-toxischer Reize

$2.3 \quad 2.3 .1$

Pathophysiologische Schmerzreaktion bei Entzündung der Pulpa aufgrund von physikalischen Reizen thermischer und/oder mechanischer Art; bei aktinisch (Strahlen) verursachten Entzündungsreaktionen

Diagnose:

- Pulpitis aufgrund physikalischer Reize

2.3.2

Pathophysiologische Schmerzreaktion bei bereits entzündeter Pulpa durch Änderung des barometrischen Drucks Diagnosen:

- Barodontalgie (beim Tauchen, Fliegen)

- Aerodontalgie (= Höhenzahnschmerz beim Fliegen)

Dauer

Lang anhaltend (Minuten/Stunden/Tage)

Qualität

Intensität

Auslösung

„Dumpf", ,„pochend" u. a.; akut: pulssynchron klopfend (auch Qualität von „Dentinschmerz" möglich)

Mittelstark bis sehr stark

Sensibilitätstest

Variabel (intermittierender Spontanschmerz); s. auch bei „Ursache“

Positiv

Perkussionstest

Röntgen

Negativ (bei Übergang in apikale Parodontitis positiv, s. unten)

Ggf. Zahnhartsubstanzläsionen bis in Pulpanähe, periradikulärer Bereich unauffällig 
Tab. 1 Fortsetzung

\begin{tabular}{|c|c|c|}
\hline \multicolumn{2}{|l|}{ Sonderformen } & $\begin{array}{l}\text { - Komplizierte Kronen-/Wurzelfrakturen } \\
\text { - Endodontisch-parodontale Läsionen (s. 3.2.1) } \\
\text { Bemerkung:Verletzungsbedingte Läsionen und Endo-Paro-Läsionen können sowohl Pulpa- als auch } \\
\text { Parodontalschmerzen auslösen }\end{array}$ \\
\hline \multicolumn{2}{|l|}{ Therapie } & Kausal \\
\hline \multicolumn{3}{|c|}{ 3. Parodontaler Schmerz (ausgehend von Parodontalgeweben) } \\
\hline \multirow[t]{2}{*}{ Ursache } & 3.1 & $\begin{array}{l}\text { Marginales Parodont } \\
\text { 3.1.1 } \\
\text { Pathophysiologische Schmerzreaktion durch mechanische Reizung (z. B. bei der parodontalen Sondierung) } \\
\text { oder durch sterile Entzündung des Parodontiums nach mechanischem Trauma (einschließlich okklusales Trauma, } \\
\text { bruxismusbedingtes heftiges Kieferpressen/Zähneknirschen und orthodontische Kräfte) } \\
\text { Diagnose: } \\
\text { - Desmodontaler Schmerz } \\
\text { 3.1.2 } \\
\text { Pathophysiologische Schmerzreaktion durch primäre bakterielle Entzündung des Parodontiums } \\
\text { Diagnosen: } \\
\text { - Parodontaler Schmerz bei Parodontitis } \\
\text { - Parodontaler Schmerz bei nekrotisierender ulzerierender Gingivitis/Parodontitis (NUG/NUP) }\end{array}$ \\
\hline & 3.2 & $\begin{array}{l}\text { Apikales Parodont } \\
\text { 3.2.1 } \\
\text { Pathophysiologische Schmerzreaktion durch sekundäre bakterielle Entzündung des Parodontiums } \\
\text { aufgrund einer infizierten Pulpanekrose (apikale/laterale Parodontitis; apikaler/lateraler Abszess) } \\
\text { Diagnose: } \\
\text { - Parodontaler Schmerz bei apikaler Parodontitis ohne/mit Abszessbildung } \\
\text { 3.2.2 } \\
\text { Pathophysiologische Schmerzreaktion auf iatrogen erzeugte chemische Reize } \\
\text { (Gewebeirritation durch endodontische Spüllösungen, Wurzelkanalfüllmaterialien etc.) } \\
\text { Diagnose: } \\
\text { Parodontaler Schmerz bei chemisch induzierter apikaler Gewebereizung }\end{array}$ \\
\hline \multirow[t]{4}{*}{ Dauer } & 3.1.1 & Lang anhaltend (Stunden/Tage) \\
\hline & 3.1 .2 & Lang anhaltend (Stunden/Tage) \\
\hline & 3.2 .1 & Lang anhaltend (Stunden/Tage) \\
\hline & 3.2 .2 & Lang anhaltend (Stunden/Tage) \\
\hline \multirow[t]{4}{*}{ Qualität } & 3.1 .1 & „Dumpf",,,drückend",,,spitz" \\
\hline & 3.1 .2 & „Dumpf",„,ziehend“,,„pochend" \\
\hline & 3.2.1 & "Scharf", „, dumpf", \\
\hline & 3.2 .2 & ${ }_{\text {"Scharf }}{ }_{, \prime \prime}$ dumpf ${ }^{\prime \prime}$, pochend" \\
\hline \multirow[t]{4}{*}{ Intensität } & 3.1 .1 & Leicht bis mittelstark \\
\hline & 3.1 .2 & Leicht bis sehr stark \\
\hline & 3.2 .1 & Mittelstark bis sehr stark \\
\hline & 3.2 .2 & Mittelstark bis sehr stark \\
\hline \multirow[t]{4}{*}{ Auslösung } & 3.1 .1 & Auf äußeren mechanischen Reiz \\
\hline & 3.1 .2 & Auf äußeren adäquaten Reiz oder spontan \\
\hline & 3.2 .1 & Auf äußeren mechanischen oder thermischen (Wärme) Reiz oder spontan \\
\hline & 3.2 .2 & Auf via Wurzelkanal wirkenden chemischen Reiz \\
\hline \multirow[t]{4}{*}{ Sensibilitätstest } & 3.1 .1 & Positiv \\
\hline & 3.1 .2 & Positiv \\
\hline & 3.2.1 & $\begin{array}{l}\text { Negativ bei apikaler Parodontitis } \\
\text { (cave:„Restsensibilität“ der Pulpa, Variabilität von Befunden bei mehrwurzeligen Zähnen u. a.) }\end{array}$ \\
\hline & 3.2 .2 & Negativ \\
\hline \multirow[t]{4}{*}{ Perkussionstest } & 3.1.1 & Positiv \\
\hline & 3.1 .2 & Positiv \\
\hline & 3.2.1 & Positiv \\
\hline & 3.2 .2 & Positiv \\
\hline \multirow[t]{4}{*}{ Röntgen } & 3.1 .1 & Unauffällig oder Verbreiterung des Parodontalspaltes \\
\hline & 3.1 .2 & Unauffällig oder vertikaler/horizontaler Knochenabbau, ggf. Verbreiterung des Parodontalspaltes \\
\hline & 3.2.1 & Unauffällig oder periradikuläre Radioluszenzen \\
\hline & 3.2 .2 & Unauffällig oder periradikuläre Radioluszenzen \\
\hline
\end{tabular}


Tab. 1 Fortsetzung

\begin{tabular}{|c|c|c|}
\hline \multicolumn{2}{|l|}{ Sonderformen } & $\begin{array}{l}\text { - Komplizierte Kronen-/Wurzelfrakturen } \\
\text { - Luxationsverletzungen } \\
\text { - Endodontisch-parodontale Läsionen (s. 3.2.1) } \\
\text { Bemerkung:Verletzungsbedingte Läsionen und Endo-Paro-Läsio } \\
\text { Parodontalschmerzen auslösen }\end{array}$ \\
\hline \multicolumn{2}{|l|}{ Therapie } & Kausal \\
\hline \multicolumn{3}{|c|}{ 4. Alveolär-ossärer Schmerz } \\
\hline \multirow[t]{3}{*}{ Ursache } & 4.1 & Physiologische Schmerzreaktion nach Zahnextraktionen oder the \\
\hline & 4.2 & $\begin{array}{l}\text { Pathophysiologische Schmerzreaktion durch bakterielle Entzünd } \\
\text { Diagnosen: } \\
\text { - Dolor post extractionem } \\
\text { - Dentitio difficilis }\end{array}$ \\
\hline & 4.3 & $\begin{array}{l}\text { Pathophysiologische Schmerzreaktion aufgrund von Knochennel } \\
\text { kauter oder nach Diffusion von endodontischen Einlagen [Arsen, } \\
\text { knochen } \\
\text { Diagnose: } \\
\text { - latrogen bedingte Knochennekrose }\end{array}$ \\
\hline \multicolumn{2}{|l|}{ Dauer } & Lang anhaltend (Tage) \\
\hline \multicolumn{2}{|l|}{ Qualität } & "Ziehend", ${ }_{, \prime}$ dumpf", „,pochend" u. a. \\
\hline \multirow[t]{3}{*}{ Intensität } & 4.1 & Schwach bis sehr stark \\
\hline & 4.2 & Mittelstark bis sehr stark \\
\hline & 4.3 & Mittelstark bis sehr stark \\
\hline \multirow[t]{3}{*}{ Auslösung } & 4.1 & Auf einen äußeren Reiz \\
\hline & 4.2 & Spontan und/oder auf äußeren adäquaten Reiz (z. B. Berührung) \\
\hline & 4.3 & Spontan \\
\hline \multicolumn{2}{|l|}{ Sensibilitätstest } & - \\
\hline \multicolumn{2}{|l|}{ Perkussionstest } & - \\
\hline \multirow[t]{3}{*}{ Röntgen } & 4.1 & \\
\hline & 4.2 & - \\
\hline & 4.3 & Unauffällig oder Aufhellung \\
\hline Therapie & & Kausal \\
\hline
\end{tabular}

5. Formenkreis atypischer Zahnschmerzen (atypische Odontalgie/Phantomzahnschmerz) Ursache

Pathophysiologische Schmerzreaktion bei Schädigungen respektive Dysfunktionen peripherer oder zentraler trigeminaler Nervenstrukturen (klinisch keine pathologisch-organischen Veränderungen feststellbar) Diagnose:

- Atypische Odontalgie (falls Zahn nicht mehr vorhanden auch: Phantomzahnschmerz)

Dauer Kurz bis lang anhaltend (Minuten/Stunden, meist kontinuierlich)

Qualität Intensität

Auslösung

Sensibilitätstest

Perkussionstest

Röntgen

Sonderform

Therapie

"Dumpf", ,, bohrend“", ,'ziehend"; kann von Zahn zu Zahn bzw. von zahnlosem Areal zu zahnlosem Areal wandern Leicht bis stark (variabel)

Spontan

Falls Zahn noch vorhanden (vgl. Phantomschmerz): positiv

Falls Zahn noch vorhanden (vgl. Phantomschmerz): negativ

Unauffällig

Neuropathischer, heterotoper Schmerz (s. 6.)

Symptomatisch (medikamentös; schmerzbezogene psychologische Therapie)

\section{otoper) Schmerz}

Pathophysiologische Schmerzreaktion bei Fehllokalisation der Schmerzquelle. (Konvergenz/Divergenz primärnozizeptiver Neuronen auf sekundär-nozizeptive Neuronen im Nucleus spinalis (Subnucleus caudalis). Primäre Afferenzen aus dem Läsionsgebiet divergieren auf Neuronen benachbarter Muskelregionen oder anderer Strukturen. Bei starken Schmerzreizen werden diese üblicherweise synaptisch ineffektiven Verzweigungen "durchgängig" und erregen diese angrenzenden Neuronen ebenfalls, sodass „der Schmerzfunke auf benachbarte Neuronen überspringen kann"

Der Schmerz wird in den Zähnen wahrgenommen (= Schmerzort); sein Ursprung (= Schmerzquelle) kann odontogen (6.1) oder nichtodontogen sein (6.2)

Diagnosen:

- Heterotoper Zahnschmerz odontogenen Ursprungs

- Heterotoper Zahnschmerz nichtodontogenen Ursprungs 


\begin{tabular}{|c|c|c|}
\hline \multirow{2}{*}{\multicolumn{2}{|c|}{$\begin{array}{r}6.1 \\
6.2\end{array}$}} & $\begin{array}{l}\text { Schmerzquelle odontogenen Ursprungs: } \\
\text { - Neuropathisch (z. B. bei Pulpitis eines anderen Zahnes, Phänomen der „Ausstrahlung“) }\end{array}$ \\
\hline & & $\begin{array}{l}\text { Schmerzquelle nichtodontogenen Ursprungs: } \\
\text { - Muskulär (z. B. bei Tendomyopathie des M. masseter oder M. temporalis) } \\
\text { - Artikulär (Kiefergelenke) } \\
\text { - Neurovaskulär (z. B. bei Migräne; Cluster-Kopfschmerz) } \\
\text { - Rhinogen/sinugen (z. B. bei viraler/allergener Rhinitis; Sinusitis maxillaris) } \\
\text { - Otogen (z. B. bei Otitis media) } \\
\text { - Okulogen (z. B. bei Iritis; Iridozyklitis) } \\
\text { - Kardiogen (z. B. bei Angina pectoris) } \\
\text { - Neuropathisch (Trigeminusneuralgie) } \\
\text { - Atypisch-neuropathisch bei Aufrechterhaltung einer verletzungsbedingten zentralen Sensibilisierung } \\
\text { durch „physiologischen“ neuronalen Input aus der Peripherie } \\
\text { (z. B. aus einer Narbe). Sonderform der atypischen Odontalgie (s. 5.) } \\
\text { - Neoplastisch (Kiefergelenktumoren; Kieferhöhlentumoren) }\end{array}$ \\
\hline Dauer & & Variabel \\
\hline Qualität & & „Scharf",„dumpf“ u.a. \\
\hline Intensität & & Variabel \\
\hline Auslösung & & Reizung der odontogenen (6.1) oder nichtodontogenen (6.2) Schmerzquelle \\
\hline Sensibilitätstest & & Falls keine Wurzelkanalbehandlung erfolgte: positiv \\
\hline Perkussionstest & & In der Regel negativ (bei Sinusitis auch positiv) \\
\hline Röntgen & & Zahn unauffällig \\
\hline Therapie & & Kausal oder symptomatisch \\
\hline \multicolumn{3}{|c|}{ 7.Zahnschmerz in Zusammenhang mit primär psychosozialen Faktoren (früher:„psychogener“ Zahnschmerz) } \\
\hline Ursache & & $\begin{array}{l}\text { Nicht durch somatische Pathologie begründbare Schmerzreaktionen als psychische Störung (anhaltende somato- } \\
\text { forme Schmerzstörung, F45.40); als Symptom bei psychischen Störungen (z. B. Psychosen, Depressionen); bei } \\
\text { psychischen Belastungen und damit verbundenen somatischen (z. B. muskulär) oder kognitiv-emotionalen Pro- } \\
\text { zessen (z. B. Krankheitsangst); als chronische Schmerzstörung mit somatischen und psychischen Faktoren (F45.41) } \\
\text { Diagnosen: } \\
\text { - "Zahnungsschmerz" ohne morphologisches Korrelat bei Kleinkindern } \\
\text { - Zahnschmerz in Zusammenhang mit ... (Nennung der diagnostizierten psychischen Störung) } \\
\text { - Chronische Schmerzstörung mit somatischen und psychischen Faktoren }\end{array}$ \\
\hline Dauer & & Variabel \\
\hline Qualität & & Variabel, bei Psychosen zuweilen bizarre Schmerzerlebnisse \\
\hline Intensität & & Variabel \\
\hline Auslösung & & Nicht bekannt \\
\hline Sensibilitätstest & & Variabel \\
\hline Perkussionstest & & Variabel \\
\hline Röntgen & & Unauffällig \\
\hline Therapie & & $\begin{array}{l}\text { Multimodal/interdisziplinär bei F45.41 die Regel, oder Behandlung der psychischen Störung, falls positiv } \\
\text { diagnostiziert }\end{array}$ \\
\hline
\end{tabular}

logiebildung zum Knochengewebe erscheint es uns aber berechtigt, den „Pulpaschmerz" nicht als Eingeweideschmerz aufzufassen [11, 54]; er bleibt aber weiterhin ein tiefer somatischer Schmerz [80].

\subsection{Mikrobielle Infektion}

Mikrobielle Reize sind die häufigste Ursache für eine Pulpaentzündung und damit verbundenem Zahnschmerz. Der Hauptgrund für die bakterielle Invasion in das Pulpagewebe ist die Zahnkaries [61]; seltenere Ursachen sind unter anderem bakterielle Infektionen, die über Infrakturen,
Zahn- bzw. Zahn-Wurzel-Frakturen und über bis zum Wurzelapex sich ausdehnende Zahnfleischtaschen die Pulpa erreichen. Als Konsequenz der letztgenannten Infektion ergibt sich eine sog. retrograde Pulpitis. Zu erwähnen sind ferner Infektionsübertragungen aus der Nachbarschaft (Sinusitis, Osteomyelitis, apikale Parododontitis des Nachbarzahnes) sowie (umstritten) eine hämatogene oder lymphogene (retrograde) Streuung von Bakterien in eine entzündete Region, z. B. einen pulpitischen Zahn (Anachorese $[3,52]$ ).

An der Entstehung und Unterhaltung von Pulpaentzündungen ist eine Rei- he endogener Faktoren beteiligt, darunter proinflammatorische Zytokine, Bradykinin, Nervenwachstumsfaktor [35], Prostaglandine [58] und Neuropeptid Y $[19,22]$.

Daneben scheinen aber auch Endotoxine eine nicht unbedeutende Rolle zu spielen. Ihre höhere Konzentration im Pulpagewebe von kariösen Zähnen mit schmerzhafter, „irreversibler“ Pulpitis (im Vergleich zu tief kariösen, aber symptomlosen Zähnen) ist ein starker Hinweis dafür, dass diese Bakterienprodukte eine Rolle für die Pathogenese der Pulpitis spielen [34]. Neuere Studien haben gezeigt, 
dass trigeminale afferente Neuronen, darunter eine capsaicinsensitive Untergruppe der Nozizeptoren, TLR4- und CD14Rezeptoren exprimieren, die für die Erkennung bakterieller Produkte (z. B. Lipopolysaccharide) bedeutsam sind. Es wird gemutmaßt, dass eine endotoxinbedingte Aktivierung von TLR4 und $\mathrm{CD}_{14}$ intrazelluläre Signalkaskaden auslöst, die unter anderem über eine periphere Ausschüttung von Neuropeptiden zum „Pulpaschmerz" führt [83].

\subsection{Chemisch-toxische Reize}

Inhaltsstoffe von Restaurationsmaterialien können Pulpaentzündungen und Schmerzen verursachen. Das Risiko erhöht sich, wenn die die Zahnpulpa überdeckende Restdentinschicht extrem dünn ist oder die Pulpa ganz freiliegt [13]. Gleiches gilt für Mittel zur Devitalisierung der Pulpa („Mortalexstirpation“), wie Paraformaldehyd und (das heute nicht mehr verwendete [31]) Arsen ("Arsenschmerz" [55]).

\subsection{Physikalische Reize}

\subsubsection{Thermische/ mechanische Reize}

Ein therapeutisch bedingter „Pulpaschmerz" kann durch eine während einer Zahnpräparation erfolgte thermische und/oder mechanische Pulpareizung ausgelöst werden (Präparationstrauma, z. B. aufgrund zu geringer Wasserkühlung und/oder zu hohem Anpressdruck des Präparationsinstruments $[51,90])$. Hierbei treten die Schmerzen oft mit einigen Tagen Verzögerung auf [85].

\subsubsection{Barodontalgie}

Eine spezielle Form pulpaler Schmerzen äußert sich als „Barodontalgie“. Diese Schmerzen entstehen durch Änderungen des barometrischen Drucks, also entweder beim Tauchen oder beim Fliegen [7o]; Letztere werden auch als Aerodontalgie (Höhenzahnschmerz) bezeichnet $[20]$.

\section{Parodontaler Schmerz}

Da die kollagenen Faserbündel des Desmodonts (Lig. parodontale) eine syndes- motische Verbindung zwischen der Zahnwurzeloberfläche (Zement) und dem Alveolarknochen bewirken (Gomphosis, Articulatio dento-alveolaris) und Gomphosen $\mathrm{zu}$ den kontinuierlichen Knochenverbindungen zählen [59, 69], sind sie Teil des muskuloskelettalen Systems. Desmodontale bzw. parodontale Schmerzen werden über im Desmodontalspalt befindliche Nozizeptoren vermittelt. Sie sind für den Patienten spätestens nach mechanischer Belastung des betreffenden Zahns (z. B. durch Druck) meist gut lokalisierbar. Es handelt sich hierbei um einen tiefen somatischen muskuloskelettalen Schmerz [56, 80]. Im Vergleich zur Pulpa ist im Zahnhalteapparat die Wahrnehmung auf Schmerz (ebenso wie für nicht schmerzhafte Reize) differenzierter.

\subsection{Marginales Parodont}

Desmodontale Schmerzen sind typischerweise Überlastungsschmerzen, z. B. aufgrund eines okklusalen Traumas wegen starken Bruxismus oder einer zu hohen Restauration [75] oder Kraftausübung mittels eines festsitzenden orthodontischen Geräts. Auch Schmerzen, die durch das Eindringen einer Parodontalsonde zum Zwecke der Messung der Sulkus- bzw. Zahnfleischtaschentiefe auftreten $[14,27]$, gehören in diese Gruppe.

Im Gegensatz dazu sind bei parodontalen Schmerzen, ausgelöst durch eine Gingivitis oder Parodontitis (primäre bakterielle Entzündung [84]), auch die anderen Komponenten des Zahnhalteapparats (Alveolarknochen; Gingiva) betroffen. Bei fortgeschrittenen Parodontalerkrankungen ist der Parodontalabszess (= Taschenabszess) eine häufig vorkommende schmerzhafte Komplikation. Ein ausgeprägter parodontaler Schmerz ist auch bei nekrotisierender ulzerierender Gingivitis/Parodontitis (NUG/NUP) vorhanden. Schmerzhafte Entzündungen der parodontalen Weichgewebe können ebenso aus der Inokulation von Fremdkörpern (z. B. Getreidespelzen aus naturbelassener Nahrung) resultieren.

Parodontale Schmerzen treten ferner im Zuge zahnärztlicher Therapie des entzündeten Zahnhalteapparats auf [82].

\subsection{Apikales Parodont}

Eine infizierte Pulpanekrose kann zu einer sekundären Entzündung des Parodontiums mit Ausbildung einer (z. B.) apikalen Parodontitis führen: Eine vertikale, in fortgeschrittenen Fällen zusätzlich auch horizontale Perkussionsempfindlichkeit des betroffenen Zahns kann in diesem Fall auf eine akute (und fortgeschrittene) Entzündung im Bereich der Wurzelspitze (apikales Desmodont) hinweisen [71].

Parodontaler Schmerz in der Apikalregion eines Zahnes kann aber nicht nur durch eine Infektion (über mikrobielle Reize), sondern auch durch chemische Reize bedingt sein, die sich im Zuge von Wurzelkanalbehandlungen ergeben können $[42,67,86]$. So kann das akzidentelle Überstopfen eines Wurzelkanals mit einem Füllmaterial (z. B. Kalziumhydroxid) zu einer chemisch induzierten Entzündung und (meist nach einigen Stunden) zu empfindlichen Schmerzen führen [62]. In anderen Fällen können mit zu starkem Druck in den Wurzelkanal gepresste und das Parodont erreichende Substanzen, wie Natriumhypochlorit (Spülflüssigkeit) oder Guttapercha (Wurzelkanalfüllmaterial), zu akut einschießenden, extrem starken Schmerzen führen (mechanisch unterstützte, chemisch bedingte Irritation des periapikalen $\mathrm{Ge}$ webes; [53]).

Für die ätiologische Einordnung von unklaren Zahnschmerzen mit variabler Symptomatik ist die Kenntnis von Mischformen wichtig. So kann bei endodontisch-parodontalen Läsionen gleichzeitig ein pulpaler und ein parodontaler Schmerz vorliegen, was die Diagnostik erschwert [56].

\section{Alveolar-ossärer Schmerz}

Der alveolär-ossäre Zahnschmerz ist ein physiologischer Knochenschmerz nach therapeutischen Eingriffen wie Zahnextraktionen, Wurzelspitzenresektionen o. Ä., oder er äußert sich als entzündungsbedingter Knochenschmerz. In letzterer Form tritt er auf als Dolor post extractionem (trockene Alveole/Alveolitis sicca dolorosa/Ostitis circumscripta) oder in $\mathrm{Zu}$ sammenhang mit dem Durchbruch eines 
Hier steht eine Anzeige.

算 Springer 
verlagerten Zahns (Dentitio difficilis; Perikoronitis; [68]).

Alveolär-ossärer Zahnschmerz kann sich darüber hinaus in Zusammenhang mit einer Knochennekrose bemerkbar machen, in deren Randbereich der noch vitale Knochen superinfiziert wird. Dies kann z. B. der Fall sein nach falscher Anwendung von Elektrokauter und nach Diffusion von zur Pulpadevitalisation eingesetzten Einlagen (Arsen, [Para-]Formaldehyd) aus dem Wurzelkanal oder der Zahnkavität über zahnhalsnahe Karies bzw. undichte Restaurationen in den Kieferknochen.

\section{Atypische Odontalgie}

Große Probleme weist die Diagnostik der atypischen Odontalgie auf, weil dieses von dem Londoner Anatomen und Chirurgen John Hunter [30] im 18. Jahrhundert erstmals beschriebene Schmerzbild nach wie vor wenig bekannt ist. Es handelt sich hierbei um einen neuropathischen Schmerz [80] mit peripherer und zentraler Sensibilisierung des trigeminalen Systems [44].

Bei der atypischen Odontalgie entwickeln bleibende Zähne nach Kariestherapie, Wurzelkanalbehandlungen oder periradikulärer Chirurgie (z. B. Wurzelspitzenresektionen) sowie zahnlose Kieferregionen nach Zahnextraktionen in seltenen und nicht vorhersehbaren Fällen dumpfe, bohrende Dauerschmerzen. Es handelt sich um eine Ausschlussdiagnose; das nozizeptive System ist hierbei die Quelle der Beschwerden [2, 81]. Wenn die atypische Odontalgie bei pulpatoten Zähnen oder im Bereich eines nicht mehr vorhandenen Zahns auftritt, spricht man auch von einem „Phantomzahnschmerz“ [49].

Neuere Studienergebnisse lassen darauf schließen, dass ein erheblicher Teil der an einer atypischen Odontalgie leidenden Patienten intraoral deutliche somatosensorische Auffälligkeiten aufweist, die als Zeichen einer eingetretenen peripheren und zentralen Sensibilisierung im Trigeminusbereich (und darüber hinaus) gewertet werden [44]. Aus diesem Grund kann der quantitativen sensorischen Testung (QST) ein gewisser diagnostischer Stellenwert zukommen [73].
In einzelnen Fällen bleibt eine atypische Odontalgie bzw. ein Phantomzahnschmerz nicht auf einen mehr oder weniger eng definierten intraoralen $\mathrm{Be}$ reich beschränkt, sondern weitet sich zu einem sog. anhaltenden idiopathischen Gesichtsschmerz (früher: atypischer Gesichtsschmerz) aus [21].

\section{In die Zähne übertragener (heterotoper) Schmerz}

Besondere Aufmerksamkeit ist schließlich den in die Zähne übertragenen (heterotopen) Schmerzen zu widmen. Hier stimmen der vom Patienten angegebene Schmerzort und die Schmerzquelle nicht überein. Das Phänomen des übertragenen Schmerzes ist im Gesichtsbereich seit Langem bekannt und dokumentiert (z. B. [76, 87]). Quellen für Zahnschmerzen können beispielsweise sein:

- andere Zähne [39],

- Kiefermuskeln ([36]; M. masseter, M. temporalis, M. pterygoideus medialis, M. pterygoideus lateralis, M. digastricus venter anterior [15]),

- Kiefergelenke [88],

- Kieferhöhlen (Sinusitis maxillaris [29]),

- neurovaskuläres System (Migräne [10]),

- Ohren (Otitis media [64, 72]),

- Hirnnerven (Trigeminusneuralgie [23]),

- Nacken-Schulter-Bereich [64],

- Herz (Angina pectoris [41]),

- Neoplasien, z. B. Kiefergelenk- [40] oder Kieferhöhlentumoren [89].

Als Schlüsselindikatoren für Zahnschmerzen, die auf nichtodontogene Schmerzquellen hinweisen, können folgende Gesichtspunkte gelten [15]:

- Auftreten spontaner multipler Zahnschmerzen,

- Fehlen einer lokalen dentalen Ursache für den Schmerz,

- reizende, brennende, nicht pochende Zahnschmerzen,

- persistierende, in der Intensität nicht wechselnde Zahnschmerzen,

- persistierende, periodisch auftretende Zahnschmerzen,
- keine Schmerzsteigerung nach lokaler Provokation (z. B. Perkussion oder thermische Reize),

- kein signifikanter Schmerzrückgang nach Lokalanästhesie oder anderen begründeten zahnärztlichen Therapiemaßnahmen.

\section{Zahnschmerz in Zusammenhang mit primär psychosozialen Faktoren}

Die Mundhöhle ist zahlreichen psychosozialen Einflussfaktoren ausgesetzt. Dies äußert sich bereits im Kleinkindalter, wenn „Zahnungsschmerzen“, für die es bislang kein wissenschaftlich plausibles Erklärungsmodell gibt, zur Rede stehen.

„Psychogene" Odontalgien - früher auch als "hysterische Zahnschmerzen“ [47] bezeichnet - sind von allen Zahnschmerzformen die mit Abstand seltensten (und umstrittensten). Abgesehen von wenigen kasuistischen Darstellungen Einzelfallberichte von Zahnschmerz im Rahmen psychotischer Störungen oder nach schwerer psychischer Traumatisierung [33] - sind nahezu keine wissenschaftlichen Daten verfügbar.

Diagnostisch nicht klassifizierbare Zahnschmerzen können, müssen aber nicht "psychogen“ sein. Da der Begriff „psychogen“ ähnlich unspezifisch ist wie die Bezeichnung „somatogen“, wird er in den heute gültigen Klassifikationssystemen nicht mehr verwendet. Sind psychosoziale Faktoren positiv diagnostiziert, kann eine klinisch relevante psychische Störung wesentliche Ursache der Schmerzen sein [18]; es kann sich jedoch auch um Normvarianten handeln (erhöhte Ängstlichkeit), die im Kontext der Schmerz- und Behandlungserfahrungen eine zentrale Bedeutung erlangt haben und bei ursprünglich nozizeptiven Prozessen dann im Sinne von aufrechterhaltenden Faktoren wirksam werden. Die IASP gibt in ihrer Klassifikation des chronischen Schmerzes [50] 4 mögliche Formen von Schmerzen mit psychischem Ursprung an:

- muskulär bedingt,

- bei Psychosen,

- hysterisch-konversionsneurotischhypochondrisch,

- bei Depressionen. 
Die IASP-Klassifikation ist inhaltlich plausibel. Terminologisch ist jedoch eine Revision dringend erforderlich. In moderneren Klassifikationssystemen wie der ICD-10 (Internationale statistische Klassifikation der Krankheiten und verwandter Gesundheitsprobleme, 10. Revision) wird der hysterisch-konversionsneurotisch-hypochondrische Schmerz, wie er in der IASP-Einteilung beschrieben wird, als somatoforme Schmerzstörung eingeordnet.

Auch der Begriff „psychogen“ wurde mit der Einführung der 3. Auflage des Diagnostischen und Statistischen Manuals psychischer Störungen (DSM III [1]) durch die derzeit noch gültige Bezeichnung „somatoform“ ersetzt. In der ICD10 [16] wird chronischer Schmerz dabei als anhaltende somatoforme Schmerzstörung diagnostiziert (F45.4). Im DSMIV-TR (Diagnostisches und Statistisches Manual psychischer Störungen, 4. Version, Textrevision [66]) ist eine differenziertere Diagnosestellung möglich: die „Schmerzstörung in Verbindung mit Psychischen Faktoren“ (DSM-IV 307.80) sowie die „Schmerzstörung in Verbindung mit sowohl Psychischen Faktoren wie einem medizinischen Krankheitsfaktor" (DSM-IV 307.89). Diese Differenzierung trägt der Tatsache Rechnung, dass eine dualistische Aufteilung von Schmerz in somatisch oder psychisch bedingt wissenschaftlich fragwürdig ist. Zudem können bei ursprünglich eindeutig durch somatische Ursachen entstandenen Schmerzen zunehmend psychische Einflüsse als wesentliche aufrechterhaltende Faktoren Bedeutung haben. Die aktuell gültigen Klassifikationssysteme für psychische Störungen (DSM-IV-TR und insbesondere ICD-10) werden derzeit überarbeitet. Dabei wird v. a. die Gruppe der somatoformen Störungen einer strikten Revision unterzogen. In der ab 2009 geltenden Fassung der ICD-10 in Deutschland (ICD10 GM) wurde für Schmerz bereits eine Revision vorgenommen, die der Differenzierung von Schmerz nach dem DSMIV entspricht [17]. Zusätzlich zur somatoformen Schmerzstörung (neue Ziffer F45.40) wird die chronische Schmerzstörung mit somatischen und psychischen Faktoren (F45.41) eingeführt. Die Kriterien für diese Diagnose sind wie folgt festgelegt:
- Im Vordergrund des klinischen Bildes stehen seit mindestens 6 Monaten bestehende Schmerzen in einer oder mehreren anatomischen Regionen, die ihren Ausgangspunkt in einem physiologischen Prozess oder einer körperlichen Störung haben.

- Psychischen Faktoren wird eine wichtige Rolle für Schweregrad, Exazerbation oder Aufrechterhaltung der Schmerzen beigemessen, jedoch nicht die ursächliche Rolle für deren Beginn.

- Der Schmerz verursacht in klinisch bedeutsamer Weise Leiden und Beeinträchtigungen in sozialen, beruflichen oder anderen wichtigen Funktionsbereichen.

- Der Schmerz wird nicht absichtlich erzeugt oder vorgetäuscht (wie bei der vorgetäuschten Störung oder Simulation). Schmerzstörungen insbesondere im Zusammenhang mit einer affektiven, Angst-, Somatisierungsoder psychotischen Störung sollen hier nicht berücksichtigt werden.

Kombinationen mit allen anderen Zahnschmerzformen und Einflüsse auf Schmerzverhalten, Schmerzschilderung und Erleben sind wahrscheinlich und mit diagnostisch-therapeutischen Problemen verbunden. Daher sollte die Diagnose Zahnschmerz in Zusammenhang mit primär psychosozialen Faktoren immer erst nach Aus- bzw. Einschluss aller anderen Odontalgien und grundsätzlich gemeinsam mit einem Psychiater bzw. Psychotherapeuten gestellt werden [65].

Es ist - wie oben bereits erwähnt durchaus möglich, dass ein Zahn mehr als eine Schmerzdiagnose aufweisen kann. Beispiele sind:

- desmodontaler Schmerz plus Pulpaschmerz (z. B. bei zu starker Zahnbewegung im Rahmen einer orthodontischen Behandlung),

- Dentinschmerz plus parodontaler Schmerz (z. B. bei der mechanischen Bearbeitung von Wurzeloberflächen im entzündeten Zahnhalteapparat),

- Pulpaschmerz plus parodontaler Schmerz (z. B. bei kombiniert endodontisch-parodontaler Läsion).

\section{Fazit für die Praxis}

Die vorgeschlagene Klassifikation erscheint uns geeignet, die verschiedenen Formen der Odontalgien differenzierter darzustellen, als dies mit den bisherigen Einteilungen der Fall war. Ihre Brauchbarkeit bzw. Überlegenheit gegenüber anderen Klassifikationen wird sich in der Praxis erweisen müssen.

\section{Korrespondenzadresse \\ Prof. Dr. med. dent. J.C. Türp}

Klinik für Rekonstruktive Zahnmedizin und Myoarthropathien, Universitätskliniken für

Zahnmedizin, Universität Basel

Hebelstr. 3, 4056 Basel

Schweiz

jens.tuerp@unibas.ch

Interessenkonflikt. Der korrespondierende Autor gibt an, dass kein Interessenkonflikt besteht.

\section{Literatur (Auswahl)}

2. Baad-Hansen L (2008) Atypical odontalgia - pathophysiology and clinical management. J Oral Rehabil 35:1-11

15. Leeuw R de (ed) (2008) Orofacial pain: guidelines for assessment, diagnosis and management, 4th edn. Quintessence, Chicago, pp 112

22. Gibbs JL, Hargreaves KM (2008) Neuropeptide YY1 receptor effects on pulpal nociceptors. J Dent Res 87:948-952

24. Goes PS, Watt R, Hardy RG, Sheiham A (2007) The prevalence and severity of dental pain in 14-15 year old Brazilian schoolchildren. Community Dent Health 24:217-224

35. Khan AA, Hargreaves KM (2007) Dental pain. Pathophysiology and management. In: Türp JC, Sommer C, Hugger A (eds) The Puzzle of Orofacial Pain Integrating Research Into Clinical Management. Karger, Basel, pp 75-90

39. Koyess E, Fares M (2006) Referred pain: a confusing case of differential diagnosis between two teeth presenting with endo-perio problems. Int Endod J 39:724-729

41. Kreiner M, Okeson JP, Michelis V et al (2007) Craniofacial pain as the sole symptom of cardiac ischemia: a prospective multicenter study. J Am Dent Assoc 138:74-79

42. Levin L, Amit A, Ashkenazi M (2006) Post-operative pain and use of analgesic agents following various dental procedures. Am J Dent 19:245-247

44. List T, Leijon G, Svensson P (2008) Somatosensory abnormalities in atypical odontalgia: a case-control study. Pain 139:333-341

48. Magnusson I, Jeffcoat MK, Donaldson D et al (2004) Quantification and analysis of pain in nonsurgical scaling and/or root planing. J Am Dent Assoc 135:1747-1754

52. Nair PNR (2006) Pathobiology of primary apical periodontitis. In: Cohen S, Hargreaves KM (eds) Pathways of the Pulp. 9th edn. Mosby, St. Louis, pp 541-579

56. Okeson JP (2005) Bell's orofacial pains. The clinical management of orofacial pain, 6th edn. Quintessence, Chicago 
57. Ommerborn M, RaabW (2006) Odontalgie. In: Hugger A, Göbel H, Schilgen M (Hrsg) Gesichtsund Kopfschmerzen aus interdisziplinärer Sicht Evidenz zur Pathophysiologie, Diagnostik und Therapie. Springer, Heidelberg Berlin New York, S 51-63

58. Patwardhan AM, Vela J, Farugia J et al (2008) Trigeminal nociceptors express prostaglandin receptors. J Dent Res 87:262-266

62. Risso PA, Cunha AJ, Araujo MC, Luiz RR (2008) Postobturation pain and associated factors in adolescent patients undergoing one- and two-visit root canal treatment. J Dent 36:928-934

63. Roh BD, Lee YE (2006) Analysis of 154 cases of teeth with cracks. Dent Traumatol 22:118-123

64. Rossman LE, Hasselgren G, Wolcott JF (2006) Diagnosis and management of orofacial dental pain emergencies. In: Cohen S, Hargreaves KM (eds) Pathways of the pulp, 9th edn. Mosby, St. Louis, pp 40-58

65. Sarlani E, Balciunas BA, Grace EG (2005) Orofacial Pain - Part II: Assessment and management of vascular, neurovascular, idiopathic, secondary and psychogenic causes. AACN Clin Issues 16:347-358

67. Sathorn C, Parashos P, Messer H (2008) The prevalence of postoperative pain and flare-up in singleand multiple-visit endodontic treatment: a systematic review. Int Endod J 41:91-99

70. Sengstbratl M, Schmid-Schwap M, Brucker C et al (2002) Barodontalgie in der Zivilluftfahrt - Eine Untersuchung des Flugpersonals der Austrian Airlines (AUA). Stomatol 99(2):31-35

71. Sigurdsson A (2008) Clinical manifestation and diagnosis. In: Ørstavik D, Pitt Ford T (eds) Essential endodontology, 2nd edn. Blackwell Munksgaard, Oxford, pp 235-258

73. Svensson P, Baad-Hansen L, Thygesen T et al (2004) Overview on tools and methods to assess neuropathic trigeminal pain. J Orofac Pain 18:332-338

77. Tronstad L (2003) Clinical endodontics. Thieme, Stuttgart

79. Türp JC, Schindler HJ, Staehle HJ (2003) Vorschlag einer aktualisierten Klassifikation von Zahnschmerzen. 127. Jahrestagung der Deutschen Gesellschaft für Zahn-, Mund- und Kieferheilkunde, gemeinsam mit dem Interdisziplinären Arbeitskreis Zahnärztliche Anästhesie, der Akademie Praxis und Wissenschaft und der LZK Nordrhein. Aachen

80. Türp JC, Hugger A, Schindler HJ (2004) Praxisnahe diagnostische Klassifikation orofazialer Schmerzen. Schweiz Monatsschr Zahnmed 114:459-466

81. Türp JC (2005) Die atypische Odontalgie. Schweiz Monatsschr Zahnmed 115:1006-1011

82. Steenberghe D van, Garmyn P, Geers L et al (2004) Patients experience of pain and discomfort during instrumentation in the diagnosis and non-surgical treatment of periodontitis. J Periodontol 75:14651470

83. Wadachi R, Hargreaves KM (2006) Trigeminal nociceptors express TLR-4 and CD14: a mechanism for pain due to infection. J Dent Res 85:49-53

87. Wright EF (2000) Referred craniofacial pain patterns in patients with temporomandibular disorder. J Am Dent Assoc 131:1307-1315

88. Wright EF (2008) Pulpalgia contributing to temporomandibular disorder-like pain: a literature review and case report. J Am Dent Assoc 139:436-440

Das vollständige Literaturverzeichnis ...

... finden Sie in der html-Version dieses Beitrags im Online-Archiv auf der Zeitschriftenhomepage www.DerSchmerz.de

\section{Somatoforme Schmerzen unter Depressionen}

Patienten mit einer Depression leiden häufiger und stärker unter Schmerzen, die sich nicht bzw. nicht vollständig mit einer organischen Grundlage begründen lassen, als Patienten ohne Depressionen. Dabei sind Frauen deutlich stärker von Depressionen und auch von den so genannten somatoformen Schmerzen betroffen als Männer. Im Rahmen einer Doktorarbeit wurden 308 Patienten über ihre Gesundheit, Schmerzsymptome, Krankheitsängste, ihr Verhalten im Krankheitsfall, die soziale Unterstützung, psychische Belastungen und viele andere Parameter befragt. Anschließend wurden die Schmerzsymptome beurteilt.

Somatoforme Symptome, auch als medizinisch nicht bzw. nicht vollständig erklärte Symptome bezeichnet, sind ein erstaunlich weit verbreitetes Phänomen. In den allgemeinärztlichen Praxen sind bis zu 80 Prozent der Symptome somatoform. Das heißt allerdings nicht, dass sich Patienten diese Symptome „einbilden“. Somatoforme Symptome werden wahrgenommen, beeinträchtigen die Lebensführung und verursachen mitunter klinisch relevantes Leid, das eine Psychotherapie, etwa eine kognitive Verhaltenstherapie rechtfertigt.

Zum Krankheitsbild einer somatoformen

Störung, die im Volksmund fälschlicherweise oft als "Hypochondrie" bezeichnet wird, gehören neben Schmerzsymptomen oftmals auch Symptome wie Schwindel, Missempfindungen in unterschiedlichen Körperbereichen, aber auch Erschöpfungsgefühle oder Müdigkeit. Wichtig, ist jedoch, dass nicht jeder, der somatoforme Symptome aufweist, auch eine diagnostizierte somatoforme Störung hat. Dies hängt vor allem davon ab, welche Beeinträchtigung in der Lebensführung bzw. welches psychische Leid für den Patienten gegeben ist.

In der Studie wurde der Frage nachgegangen, welchen Einfluss Depressionen auf das Schmerzerleben von Patienten haben und ob sich dies unterscheidet, wenn es sich um medizinisch nicht erklärte Schmerzen beziehungsweise um medizinisch erklärte Schmerzen handelt. Das Ergebnis weist daraufhin, dass bei Patienten, die aktuell unter einer Depression leiden oder in den letzten zwölf Monaten davon betroffen waren, die Anzahl der somatoformen Schmerzen in unterschiedlichen Körperbereichen wesentlich höher ist als bei Patienten ohne Depressionen. Aus den Ergebnissen könnte auch der Rückschluss gezogen werden, dass Menschen, die mit einer Vielzahl von Schmerzen in die Hausarztpraxis kommen, die nicht vollständig medizinisch erklärt sind, mit höherer Wahrscheinlichkeit eine behandlungsbedürftige Depression aufweisen.

Welche Bedeutung die richtige Einordnung und Beurteilung der Schmerzerkrankungen im Hinblick auf das Gesundheitswesen hat, zeigt das Ergebnis der Studie, wonach 73 Prozent der Schmerzen von den Hausärzten als somatoform beurteilt wurden und sich demgegenüber nur 27 Prozent medizinisch vollständig begründen ließen.

Im Falle der organisch erklärten Schmerzen macht es auch keinen Unterschied, ob die Patienten unter einer Depression leiden oder nicht: Anzahl, Dauer und Beeinträchtigung durch die Schmerzen sind hier in beiden Patientengruppen in etwa gleich.

Quelle:

Johannes Gutenberg-Universität Mainz, www.uni-Mainz.de 